\title{
Stage IIIA Prostate Cancer AJCC v8
}

National Cancer Institute

\section{Source}

National Cancer Institute. Stage IIIA Prostate Cancer A/CC v8. NCI Thesaurus. Code C140170.

Stage IIIA includes: T1-2, N0, M0, PSA 20 or more, Grade Group 1-4; T1: Prostate cancer with clinically inapparent tumor that is not palpable. T2: Prostate cancer in which the tumor is palpable and confined within the prostate. NO: Prostate cancer with no positive regional nodes. M0: Prostate cancer without evidence of distant metastasis. Grade Group 1: Gleason Score 6 or less, Gleason Pattern 3 or less+3. Grade Group 2: Gleason Score 7, Gleason Pattern 3+4. Grade Group 3: Gleason Score 7, Gleason Pattern 4+3. Grade Group 4: Gleason Score 8, Gleason Pattern 4+4. (AJCC 8th ed.) 